\title{
Zum 10. Todestag von Erwin H. Ackerknecht (1906-1988)
}

Hans H. Walser

Am 18. November 1998 jährte sich der Todestag von Erwin H. Ackerknecht zum zehnten Male. Grund genug, dieses ausserordentlichen Mannes zu gedenken, der wie kein anderer die Medizingeschichte in unserem Land geprägt hat. Er war der erste, der hierzulande seine ganze Kraft als Arzt und Historiker dieser Wissenschaft widmen konnte. Er hat dies reichlich vergolten, indem er der Medizingeschichte in der Schweiz Offenheit, Glanz und Ausstrahlung verlieh und mit seinem internationalen Ansehen Zürich zu einem Mittelpunkt medizinhistorischer Forschung und Lehre machte.

Erwin H. Ackerknecht wurde am 1. Juni 1906 in Stettin im damaligen Vorpommern geboren. Sein Vater Erwin Ackerknecht (1880-1960) war dort Direktor der städtischen Bibliothek; er hatte einen guten Namen als Autor, Literaturkritiker und als Förderer junger Talente. Viele junge Schriftsteller gingen bei ihm ein und aus, von denen manche später berühmt wurden, wie z.B. Hermann Hesse. So wuchs Erwin zusammen mit seiner Schwester in einer lebhaften, bürgerlich-intellektuellen Atmosphäre auf. Stettin wurde für ihn so zur Heimatstadt, das Haus der Eltern aber war rein schwäbisch; schwäbisch fühlte und sprach man, und selbst die Angestellten des Hauses stammten aus Württemberg. Nach dem Abitur wollte Erwin Kunstmaler werden, doch widersetzte sich der Vater diesem Wunsch, und so wählte der junge Mann Medizin als Studium.

Erwin Ackerknecht begann sein Studium 1924 in Freiburg im Breisgau. Er studierte mit grosser Begeisterung - «I enjoyed studying tremendously» schrieb er später - jedoch nicht nur Medizin, sondern auch Ökonomie, Kunstgeschichte und Literatur. Er fand gar einmal, das Medizinstudium sei «wie eine Theateraufführung» an ihm vorbeigegangen, wirklich ernst geworden sei es erst vor dem Schlussexamen. Lag es wohl an seinen akademischen

Prof. Dr. Hans H. Walser, Mühlebachstrasse 82, CH-8008 Zürich 
Lehrern, wenn er trotzdem so wohlfundierte, solide medizinische Kenntnisse hatte? Von diesen Lehrern sind allerdings viele in die Geschichte eingegangen. So etwa in Freiburg Lexer und Trendelenburg, in Kiel v. Moellendorff und Höber, in Berlin der Internist Kraus, dann Lubarsch, Bracht wie auch A. Bier, und schliesslich in Wien Eiselsberg, Hochenegg, Sternberg, Wagner-Jauregg und Wenckebach. Er promovierte 1931 in Leipzig mit einer Dissertation über die Medizinalreform von 1848 bei Henry E. Siegrist.

Er war dann Assistenzarzt auf der internen Klinik in Leipzig bei Assmann und bei Morawitz. Er rechnete es Morawitz hoch an, dass er ihn anstellte, obwohl man ihn vor dem «Kommunisten Ackerknecht» gewarnt hatte er war inzwischen als linker Studentenpolitiker bekannt. Er erachtete die praktisch-ärztliche Tätigkeit gerade für einen Medizinhistoriker als sehr wichtig, da sie ihm Nähe zum «Zielpublikum» der Studenten und Ärzte verschaffte. Vom Januar 1932 an setzte er diese Tätigkeit an der psychiatrischen Klinik in Berlin-Buch fort. Nun aber ereilte ihn sein politisches Schicksal, und die Politik bestimmte den weiteren Lebenslauf.

Politik lag ihm im Blut, der Zeitgeist gab den Ausschlag für den Kommunismus. «I was ... an enthusiastic addict of Marxism» sollte er später ironisch sagen. Von der Jugendorganisation der Kommunistischen Partei enttäuscht, wandte er sich um so eifriger Trotzki zu. Von diesem wurde er zuerst in Wien, dann in Berlin mit organisatorischen und agitatorischen Aufgaben betraut. Mit dem Sieg des Nationalsozialismus wurde die politische Arbeit gefährlich. Um Haaresbreite entging er, zusammen mit dem Sohn Trotzkis, der Verhaftung. Nach einem Aufenthalt bei Trotzki in der Türkei kam er in Paris an.

Nach weiteren Enttäuschungen löste er sich immer mehr vom politisch praktizierten Kommunismus, aber am kommunistischen Glauben hielt er noch lange fest. So schreibt Heinz Abosch, ein Mitemigrant, wie Dr. Ackerknecht sich 1937 letztlich mit der Hoffnung tröstete: «Irgendwo auf der Welt wächst vielleicht ein neuer Lenin heran, der die Lösung bringen wird.» Nach der Beerdigung von Trotzkis Sohn, der wahrscheinlich ermordet worden war, wandte er sich angewidert definitiv von der Politik ab. Die Liste seiner Weggefährten, die entweder in Nazideutschland oder in der Sowjetunion ermordet wurden, ist lang.

Ungebrochen aber war sein Lebenswille: So fand er in Paris bei allen Mühen und Sorgen des Emigrantendaseins doch Zeit, am Musée de l'homme Ethnologie zu studieren, was ihm später sehr zustatten kam.

Der Ausbruch des Krieges brachte ihm zunächst die Gefangenschaft als staatenloser Ausländer, dann den Dienst in einer Pioniergruppe, mit der er vor der deutschen Wehrmacht nach Süden floh. Es folgte eine äusserlich ruhige Zeit in Aix-en-Provence in angstvoller Erwartung des Visums für die 
Vereinigten Staaten. Dieses traf schliesslich ein, und nach einer abenteuerlichen Fahrt erreichte er 1941 New York.

Amerika empfing den Emigranten keineswegs mit offenen Armen. Die erste Zeit war hart, eben ein gefährdetes, ungewisses Emigrantendasein. Die Rettung kam durch eine Anstellung bei Henry E. Siegrist, dem Leipziger Lehrer, der inzwischen an der Johns Hopkins Universität in Baltimore Medizingeschichte lehrte. Siegrist stand damals auf der Höhe seines Ruhmes, sein Porträt sah man in der Presse, seine Stimme hörte man am Radio, man suchte ihn als Redner, man wünschte seine Kommentare. Für seinen Schüler hatte er wenig Zeit. Dieser machte sich inzwischen mit der Sichtung ethnologischer Literatur für ein Buch des Chefs nützlich. Der eigentliche Lehrer Ackerknechts wurde Owsei Temkin. Aber wollte Ackerknecht ein kleiner, schlecht bezahlter Angestellter des Instituts bleiben? Nein, es galt weiterzukommen.

Das American Museum of Natural History in New York bot im Januar 1945 dazu eine Möglichkeit. Der Direktor, Harry L. Shapiro, wurde für Ackerknecht zum Vorbild. Nicht genug konnte er die Möglichkeiten des Museums und die Qualität der wissenschaftlichen Arbeit loben - und er lobte selten. Mitarbeiterinnen waren die damals weltberühmten Ethnologinnen Margaret Mead und Ruth Benedict, als Besucher fanden sich fast alle Ethnologen ein, die wissenschaftlich zählten. Ackerknecht war auf dem besten Weg, bei der Ethnologie zu bleiben. Auch seine Publikationen deuteten in diese Richtung.

Der Entscheid über die Zukunft fiel im Januar 1947 mit der Berufung als Professor für Medizingeschichte an die Universität von Wisconsin in Madison. Der dortige Dekan, W. S. Middleton, schuf ideale Arbeitsbedingungen, und das offene freundliche Entgegenkommen der Mediziner wie auch der Historiker und der Ethnologen sagten Ackerknecht sehr zu.

In diesen Jahren publizierte Ackerknecht die Biographie von Rudolf Virchow, wohl sein umfangreichstes Werk, sowie die Kurze Geschichte der Medizin, sein weitaus erfolgreichstes Buch. Reisen nach Europa liessen aber den Wunsch nach Forschungsmöglichkeiten wach werden, wie sie nur auf dem alten Kontinent möglich waren. Zudem begann sich das FBI in jener McCarthy-Zeit für seine politische Vergangenheit zu interessieren, und mit dem Rücktritt von W. S. Middleton hatten sich die Arbeitsbedingungen verschlechtert. So nahm Ackerknecht gerne den Ruf nach Zürich an, wo er 1957 eintraf. Das Verdienst der Berufung fällt vor allem dem Pharmakologen Hans Fischer zu. Zürich war für Ackerknecht ein Glücksfall: Das einzige deutschsprachige medizinhistorische Institut in einem nicht von Nazideutschland zuvor besetzten Land. Wie schwierig die Verhältnisse in der Bundesrepublik 
Deutschland geworden wären, sollte sich bald zeigen. Die Berufung war aber auch für die Universität Zürich ein Glück: Sie erhielt einen international anerkannten Vertreter der Medizingeschichte. Diese hatte bisher ein Schattendasein geführt: Henry E. Siegrist hatte sich zwar 1921 hier habilitiert, war aber bald anderwärts seiner Karriere gefolgt. Gustav A. Wehrli hatte zwar die grosse Sammlung begründet, doch er fand bei Studenten und Ärzten wie dann auch der gelehrte Bernhard Milt wenig Widerhall.

Die ersten fünfzehn Jahre in Zürich waren für Ackerknecht eine glückliche Zeit. Er hatte nun alles, was die Essenz seines Lebens ausmachte: ein schönes Institut und eine grosse Bibliothek, vorbildlich betreut von Margret Curti, Ansehen in der Fakultät, regen Zuspruch von Studenten und Ärzten, grossen Erfolg mit seiner Vorlesung. Einzelne Kollegen wie Hermann Mooser und Wilhelm Löffler waren ihm geradezu kongenial, der Freund Konrad Akert war 1962 nach Zürich zurückgekehrt.

All dies war eine gute Basis für die wissenschaftliche Tätigkeit. So zählte er selbst für jene Jahre 7 Bücher, 183 Artikel und rund 140 Dissertationen. Freude machte ihm die Berufung seiner Assistentin Esther Fischer-Homberger nach Bern.

Ackerknecht blieb zeitlebens ein «politischer Mensch», seine früheren politischen Jahre waren ihm stets gegenwärtig geblieben. 1964 wurde er auf dramatische Weise erneut mit jener Zeit konfrontiert. Etwa von 1956 an begann er versöhnlicher an Deutschland zu denken. Der Medizinhistoriker in Göttingen, G. Rath, wurde zum Freund. Zufällig erfuhr Ackerknecht, dass dieser Freund einen politisch belasteten Arzt zur Habilitation in Medizingeschichte vorgeschlagen hatte. Es zeigte sich dann, dass in einem analogen Fall ein schwer belasteter Dozent bereits akademisch tätig war. Ackerknecht reagierte wütend und heftig. Es kam zu einem internationalen Skandal, dessen Einzelheiten von Christoph Mörgeli und Anke Jobman ausführlich dargestellt worden sind. Der frühere SS-Obersturmführer A. Berg und der frühere SS-Hauptsturmführer B. J. Gottlieb fanden es schliesslich klüger, auf eine akademische Laufbahn zu verzichten.

Die Studentenunruhen von 1968 weckten Ackerknechts politische Aktivität erneut. Er befand sich diesmal auf der Seite der etablierten Mächte. Dies mag paradox erscheinen, war aber konsequent. Er verabscheute die neomarxistischen, israelfeindlichen und antiamerikanischen Parolen der krawallierenden Studenten und ermahnte die Universitätsleitung zur Festigkeit.

Diese beiden Ereignisse zeigen, wie sehr sich die politische Überzeugung Ackerknechts gewandelt hatte. Der junge, begeisterte Marxist war zum bürgerlichen Demokraten geworden. Die Wandlung war allmählich erfolgt. 
Er sagte, dass er in seiner Jugend gesehen habe, wie schlimm der Kapitalismus sei, dass er inzwischen eine noch schlimmere Ideologie erlebt habe, um zu schliessen: «Man kann eben nur das kleinere Übel wählen.» Die politische Entwicklung in Deutschland beobachtete er mit bedingter Zustimmung. Das Bundesverdienstkreuz, das ihm 1983 verliehen wurde, nahm er gleichmütig entgegen, während ihn die Zuerkennung der französischen «Palmes académiques» in helle Begeisterung versetzt hatte. Nach Deutschland wurde er zu Vorträgen nach der Affäre Berg/Rath nie mehr eingeladen; von den deutschen Medizinhistorikern hielten nur wenige zu ihm.

Die eigene Identität hat ihn oft beschäftigt. Eher überraschend sagte er einmal: «Ich bin eben doch ein Deutscher», aber er war es doch nicht mehr ganz. Amerikaner? - auch nicht, so gerne er Bürger der USA war. Frankreich stand seinem Herzen wohl am nächsten, doch ein Franzose war er gewiss nicht; «also ein Nomade» resümierte er sachlich. Die Ablehnung seines Bürgerrechtsgesuches durch die Stadt Zürich verletzte ihn tief. In seinem Innern schlummerte wohl der Wunsch nach einer wahren Heimat.

Bereits im Jahre 1971 trat er von der Professur zurück. Dies bedauerte er nachträglich, vor allem aber bedauerte er, dass er nicht sofort nach Amerika zurückkehrte. Sein Urteil über die Schweiz war: «... a good place for the foreigner as a worker, ... not much for him as a human being.» Dabei waren seine äusseren Lebensumstände unverändert gut, vor allem konnte er weiterhin forschen und publizieren, aber das Alter gefiel ihm nicht. Als ihm Owsei Temkin schrieb, wie gut er seinen Lebensabend gestaltet habe, meinte er bloss: «Old age, no, not for me.» Er starb in seinem Heim am 18. November 1988. Einige Tage später versammelten sich seine Familie und seine Freunde zu einer schlichten Trauerfeier auf dem Friedhof Manegg. Sein Freund Konrad Akert sprach Worte des Dankes und des Abschieds.

Das Werk von E. H. Ackerknecht ist sehr umfangreich, doch soll versucht werden, anhand der grossen Arbeiten die Hauptlinien seines Schaffens nachzuzeichnen.

Für den jungen Arzt und den marxistischen Politiker konnte es kaum ein passenderes Thema für die Doktorarbeit geben als die Darstellung der Reformbewegung in der deutschen Revolution von 1848. Dabei konnte die enge Verflechtung der Medizin mit den sozialen und politischen Mächten klar aufgezeigt werden.

Der unbändige Drang des deutschen Volkes nach Freiheit und Einheit des Staates, zusammen mit der Verelendung des Proletariates, hatte eine explosive Stimmung geschaffen, die sich in einer Revolution entlud. Überall aber, wo es um die Sache des Volkes ging, kämpften Ärzte an der vordersten 
Front. Besonders Rudolf Virchow, einer der Führer der Bewegung, verwies auf die sozialen Ursachen der Krankheiten - damals dachte man vor allem an die epidemischen Infektionskrankheiten -, und er formulierte: Die Medizin ist eine soziale Wissenschaft, die Politik ist Medizin im Grossen. In Preussen war nur ein Drittel der Bevölkerung medizinisch einigermassen gut versorgt, woran viele Missstände schuld waren. Besserung erwartete man von der Hebung des Ärztestandes, vom unentgeltlichen medizinischen Unterricht mit strengen, aber übersichtlichen Prüfungen, und von der Reform des Armenwesens. All diese Forderungen und Ideen liessen sich - davon waren jene Ärzte überzeugt - nur in einem freiheitlichen, einheitlichen und republikanischen Deutschland verwirklichen. Die politische Revolution, die ein solches Deutschland errichten sollte, wurde aber von den damaligen Regierungen blutig niedergeschlagen. Von den medizinischen Postulaten wurden einige im Lauf der folgenden Jahrzehnte verwirklicht, andere beschäftigen uns noch heute.

Ackerknechts nächste Arbeiten entstanden bereits in den Vereinigten Staaten:

Die Untersuchung über die Malaria im oberen Mississippi-Tal 1760-1900 zeigte wie die Arbeit über die Medizinalreform von 1848, dass soziale, wirtschaftliche und politische Ursachen am Verschwinden dieser Krankheit beteiligt waren. Es gab aber auch viele andere Faktoren. Die ärztliche Behandlung kam dabei nicht gut weg, denn der gebräuchliche Aderlass war einer Heilung gewiss nicht förderlich gewesen. Erst die allgemeine Verwendung von Chinin machte die Therapie effizienter.

Das grosse Buch über Rudolf Virchow setzte das Thema der Medizinalreform von 1848 fort: Virchow war einer ihrer wichtigsten Repräsentanten gewesen, er wollte jedoch vor allem Arzt sein. Als solcher machte er auf dem Gebiet der pathologischen Anatomie zahlreiche Entdeckungen. Vor allem aber schuf er die Zellularpathologie; sein Buch darüber von 1858 wurde zu einer Grundlage aller seitherigen medizinischen Forschung. Als Politiker stiess Virchow mit seiner Forderung nach einem nicht nur geeinten, sondern auch republikanischen Deutschland im preussischen Landtag heftig mit Bismarck zusammen. Bismarcks Politik führte schliesslich zu einem geeinten, nicht aber freiheitlichen Deutschland. Die letzten Lebensjahrzehnte widmete Virchow vor allem der Anthropologie. - Ackerknechts Buch wendet sich an Leser, die sich intensiv mit Medizingeschichte befassen, als Feierabendlektüre ist es weniger geeignet.

Grosse Verbreitung fand dagegen Ackerknechts Kurze Geschichte der Medizin, erstmals 1955 englisch erschienen. Dieses Buch hat unendlich vielen Studenten und Ärzten einen ersten Zugang zur Geschichte der Medizin 
eröffnet. Ackerknecht gliederte den Stoff übersichtlich nach Epochen und gab so dem Leser einen Raster, in welchem er weitere Kenntnisse einbauen konnte. Bei den deutschen Studenten wurde das Buch als «Der kleine Ackerknecht» bekannt, eine Bezeichnung, die der Verfasser mit Schmunzeln quittierte.

Die Kurze Geschichte der Psychiatrie erschien 1957 pünktlich zum 2. Internationalen Kongress für Psychiatrie in Zürich. Ackerknecht stellt darin die Entwicklung der Psychiatrie anhand der wichtigsten Werke dar. Zwei weitere Bücher, die Geschichte und Geographie der Krankheiten und die Geschichte der Therapie erschienen 1963 bzw. 1970. Alle drei Werke enthalten viel Wissenswertes, doch mag der heutige Leser die neuen Erkenntnisse der letzten Jahrzehnte vermissen.

Zum Gültigsten, was Ackerknecht geschaffen hat, gehören seine Beiträge zur Ethnomedizin und zur Anthropologie. Die Gall'sche Schädelsammlung in Paris (im Musée de l'homme) verdankt ihm Katalog und Bearbeitung. Diese Sammlung von F. J. Gall (1758-1828) hat grosse Bedeutung für die Geschichte der Hirnforschung und der Psychiatrie. Ackerknecht mag es Genugtuung bereitet haben, dass sie knapp dem Zugriff der deutschen Besatzer von Paris entgangen war. Die wichtigsten Arbeiten zur Ethnologie erschienen 1971 in einem Sammelband. Ackerknechts ethnologische Ergebnisse haben weitgehend Anerkennung gefunden, sie lauten:

1. Die sog. «primitive Medizin» ist eigenständig und keine blosse Vorstufe zur heutigen Medizin.

2. Sie ist zur Hauptsache magisch.

3. Sie ist mehr sozial als biologisch bedingt. Die jeweilige Gesellschaft bestimmt, wer krank und wer gesund ist.

4. Die primitive Medizin wirkt sozialisierend und ist für die jeweilige Gesellschaft unabdingbar.

Das letzte Buch Ackerknechts behandelt die Medizin in Paris zwischen 1794 und 1848. Wieder geht es um eine Revolution, diesmal um die grosse Revolution von 1789, die ihr Ziel erreicht und dabei die alten medizinischen Strukturen weggefegt hatte. Erst 1794 beginnt der Aufbau einer Spitalmedizin: In 48 Spitälern wurden zehntausende Patienten behandelt. Die alte «Faculté de médecine», die noch fast wie zu Zeiten Molières funktioniert hatte, wurde durch eine «Ecole de santé» mit 12 Professuren ersetzt. Körperliche Untersuchung, Autopsie und Statistik waren die methodischen Grundpfeiler der neuen Medizin. Die Chirurgie wurde mit der Medizin vereinigt; damit hatte die letzte Stunde der mediävalen Medizin geschlagen. Die Chirurgen wurden nun sogar führend, viele ihrer Namen sind noch heute jedem Arzt geläufig (Desault, Dupuytren, Lambert, Velpeau, u.a.). 
Paris war in diesen Jahrzehnten die Hauptstadt der Weltmedizin, und so blieb es bis zur Mitte des Jahrhunderts; dann übernahm allmählich Deutschland die Führung. Mit dem Jahr 1848 beendigte Ackerknecht seine Darstellung. Die grosse Epoche der Pariser Medizin war die Zeit zwischen zwei Revolutionen.

Im Jahre 1981 feierte die «Académie de médecine» in Paris den zweihundertsten Geburtstag von Laennec. Die Sitzung war sehr feierlich und wurde eingeleitet durch eine Rede des Staatspräsidenten Giscard d'Estaing. E. H. Ackerknecht war einer der Redner. Wer wollte, konnte an den Lebenslauf und das Werk dieses Mannes denken, der fünfzig Jahre zuvor als mittelloser und gefährdeter Emigrant in dieser Stadt gelebt hatte.

\section{Literatur}

\section{Werke von E. H. Ackerknecht}

Das Verzeichnis der Werke von E. H. Ackerknecht, erstellt von Margret Curti, findet sich in GESNERUS 23,5-12 (1966) mit Nachträgen 33,3-7 (1976) und 43,6-10 (1986) sowie 45,311-312 (1988). Daraus wurden insbesondere folgende Titel erwähnt:

Beiträge zur Geschichte der Medizinalreform von 1848. Sudhoffs Archiv für Geschichte der Medizin 25, 61-109, 113-183 (1932, Neudruck Wiesbaden 1963)

Rudolf Virchow. Arzt, Politiker, Anthropologe. Stuttgart 1959 (englisch: Madison/Wisconsin 1953) Malaria in the Upper Mississippi Valley 1760-1900. Bull. Hist. Med. Suppl. 4 (Baltimore 1945)

Kurze Geschichte der Medizin, bzw. Geschichte der Medizin. Stuttgart 1959 (englisch: A Short History of Medicine. New York 1955). Zahlreiche Neuausgaben und Übersetzungen, 5. deutsche Auflage Stuttgart 1986

Medicine at the Paris Hospital 1794-1848. Baltimore 1967 (französisch: Paris 1986)

Medicine and Ethnology. Selected Essays, ed. H. H. Walser and H. M. Koelbing. Bern 1971 (spanisch: Madrid 1985)

\section{Biographische Quellen}

Ackerknecht, E.H.:Autobiographical Notes. July 1986Typescript,26 pp. (Ackerknecht wünschte ausdrücklich keine Publikation des Originaltextes)

Fischer-Homberger E.: Interview mit E. H. Ackerknecht Tonband (Medizinhistorisches Institut der Universität Zürich)

Ackerknecht E. H.: Persönliche Mitteilungen in den Jahren 1957-1988

\section{Weitere Literatur}

Fee, Elizabeth and Theodore M. Brown (ed.): Making Medical History. The Life and Times of Henry E. Siegrist. Baltimore \& London 1997

Abosch Heinz: Flucht ohne Heimkehr. Aus dem Leben eines Heimatlosen. Stuttgart 1997

Mörgeli Chr. und Anke Jobmann: Erwin H. Ackerknecht und die Affäre Berg/Rath von 1964 (Manuskript, erscheint im Jahrbuch des Robert Bosch Instituts, 1998)

Siegrist Henry E.: A History of Medicine. Volume I: Primitive and archaic medicine. New York 1951 